\title{
Simulated Energy Distribution of an Electron-Beam Irradiated on Metal-Halide Perovskite Photovoltaic Devices
}

\author{
Yu-Lin Hsu ${ }^{1}$, Kaden Powell ${ }^{1}$, Chongwen $\mathrm{Li}^{2}$, Yanfa Yan $^{2}$ and Heayoung Yoon ${ }^{1}$ \\ ${ }^{1}$ University of Utah, United States, ${ }^{2}$ University of Toledo, United States
}

Characterization techniques based on electron-beam (e-beam) probes are increasingly used for investigating inhomogeneous local properties of a wide range of semiconductor material devices. Recent studies have shown distinct microstructural properties of metal-halide perovskites using electron microscopy $[1,2]$. While powerful, there are some concerns about possible beam damage of inorganic-organic perovskites via chemical-bond breakage and local heating [3]. At a high $\mathrm{kV}(>10 \mathrm{kV})$, an e-beam injected on top of perovskite devices may reach the glass bottom substrate, potentially causing charging artifacts (e.g., Figure 1a). In this work, we present a method to estimate the e-beam energy distribution of each layer of perovskite devices based on Monte-Carlo simulations (Figure 1b) and customized MATLAB scripts. Monte-Carlo simulations have been extensively used to calculate e-beam scattering and interaction profiles in solid materials (e.g., Win X-ray [4], EISS [5], and MC-SET [6]). Among various programs, CASINO (monte CArlo SImulation of electroN trajectory in sOlids) provides rapid simulation results with a simple user interface [7]. Moreover, the resulting simulation datasets can be exported into text files for further statistical analysis. A typical CASINO model uses an array of 50 (width; $x$-axis) $\times 50$ (depth; y-axis) $\times 50$ (height; z-axis) cells, corresponding to 50 planes in any given direction of the sample. The size of each cell (i.e., width, depth, height) is determined by the simulated interaction volume that increases with an accelerated beam voltage. For example, at $1 \mathrm{kV}$, the calculated cell height (z-axis) was about $0.26 \mathrm{~nm}$, and the cell width (x-axis) was 0.39 $\mathrm{nm}$. The height and the width of this cell increase to $184 \mathrm{~nm}$ and $303 \mathrm{~nm}$ at $30 \mathrm{kV}$. Each cell in the array (50 $\times 50 \times 50$ ) contains the simulated energy density with 6 significant digits and the values down to $\approx 10-12$. Our MATLAB program stores up to 15 significant digits for all calculations. Displayed numbers typically have 3 to 4 decimal places included for better readability. The MATLAB script provides the color-coded contours of energy distribution in the $\mathrm{X}-\mathrm{Z}$ plane, which shows the same plot as seen in CASINO, with a more accurate estimation of range and diameter (Figure 2a, bottom). To calculate the percentage of e-beam energy distributed at each layer, we sum the total energy in each simulation plane and divide it by the total simulation energy. Care must be taken as the units of the CASINO data files (i.e., energy by position) do not appear to correlate to actual values. The units can be used to determine relative energy densities together with (i) the backscattered electron energy (BSE) and (ii) the expected energy from a beam for a given number of electrons. If the thickness of a layer is thinner than the unit cell height (i.e., z-direction), the energy deposition of the layer may be missed. We resolve this issue by dividing the energy from a specific $2 \mathrm{D} x-y$ plane proportionally among all constituent layers of the device. If an ultrathin layer overlaps or is contained within a specific 2D $x-y$ plane (i.e., 2D cell array) at a given z-value, the amount of energy assigned to this ultrathin layer corresponds to the percentage of the 2D x-y plane. The remaining portions of the energy are also assigned to the other materials overlapping the 2D x-y cell array. Our approach provides more accurate results than in the CASINO program, where all of the energy from a given $\mathrm{x}-\mathrm{y}$ area is assigned to a single $\mathrm{z}$ height, regardless of the material layers in that plane and their thicknesses. Figures $2 b$ and $2 c$ show the simulated energy distribution of a MAPbI3 PV device that is comprised of several thin-film layers: Au (60 nm) / Spiro (220 nm) / MAPbI3 (550 nm) / SnO2 $(35 \mathrm{~nm}) /$ ITO $(200 \mathrm{~nm}) / \mathrm{SiO} 2$ (substrate). Here, ITO is indium tin oxide, and MA is methylammonium. SpiroOMeTAD or 2,2',7,7'-Tetrakis [N, N-di(4-methoxyphenyl) amino]-9,9'- spirobifluorene is used for the holetransfer layer (HTL). The simulated energy distribution indicates that the e-beam only begins to interact with the sensitive MAPbI3 layer at about $5 \mathrm{kV}$. At a beam voltage higher than $13 \mathrm{kV}$, the e-beam has interacted with the $\mathrm{SiO} 2$ layer. At $15 \mathrm{keV}$, we estimate approximately $44 \%$ of injected energy deposited into the MAPbI3 layer and $5.5 \%$ into the $\mathrm{SiO} 2$ substrate. Our analysis suggests that the deterioration of perovskite devices 
might be related to not only chemical bonding breakage or heating, but also the charging of the glass substrate at high e-beam voltages (e.g., > $15 \mathrm{kV}$ ). Together with the CASINO simulation, our MATLAB code presented in this paper allows layer-by-layer analysis of the e-beam irradiated multilayered perovskite PV devices, permitting experiments to more conclusively decouple the e-beam effects at each layer. This work was supported by the U.S. Department of Energy's Office of Energy Efficiency and Renewable Energy (EERE) under the DE-FOA-0002064 program award number DE-EE0008985.

(a)

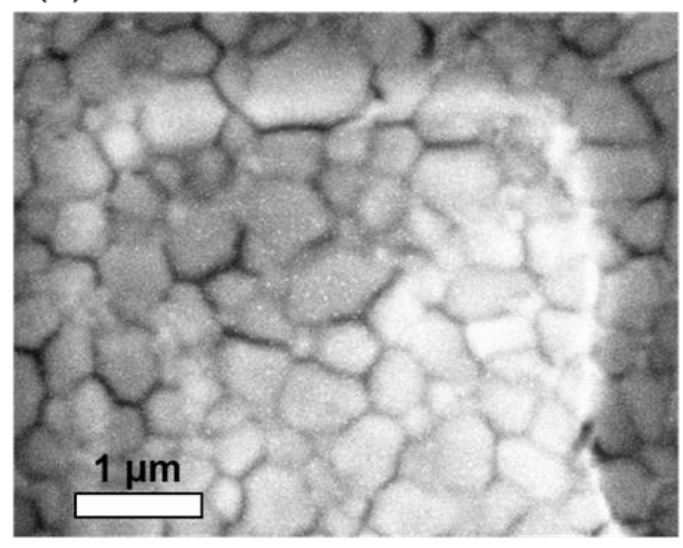

(b)

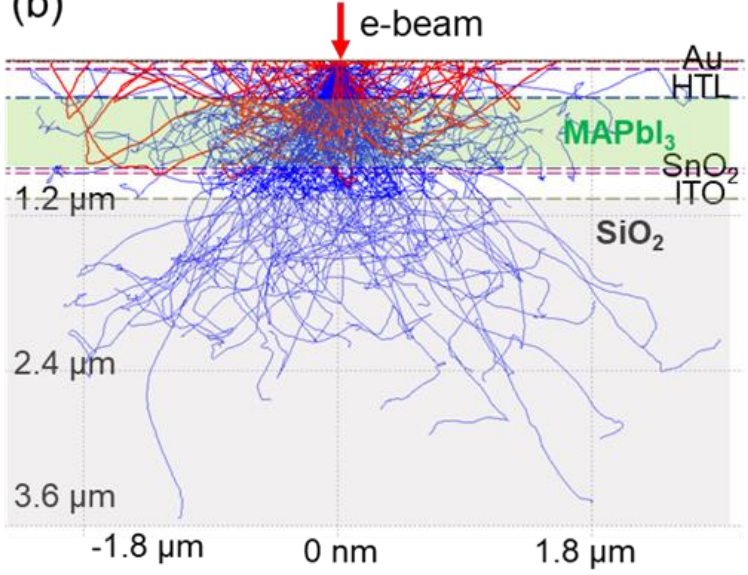

Figure 1. (a) Scanning electron microscopy (SEM) image of MAPbI3 PV device, showing a possible charging effect at the insulating SiO2 layer as indicated by brightness contrast. (b) Representative Monte Carlo e-beam simulation where lines indicate primary electron paths. Backscattered electrons (red) and electrons that remain in sample (blue).
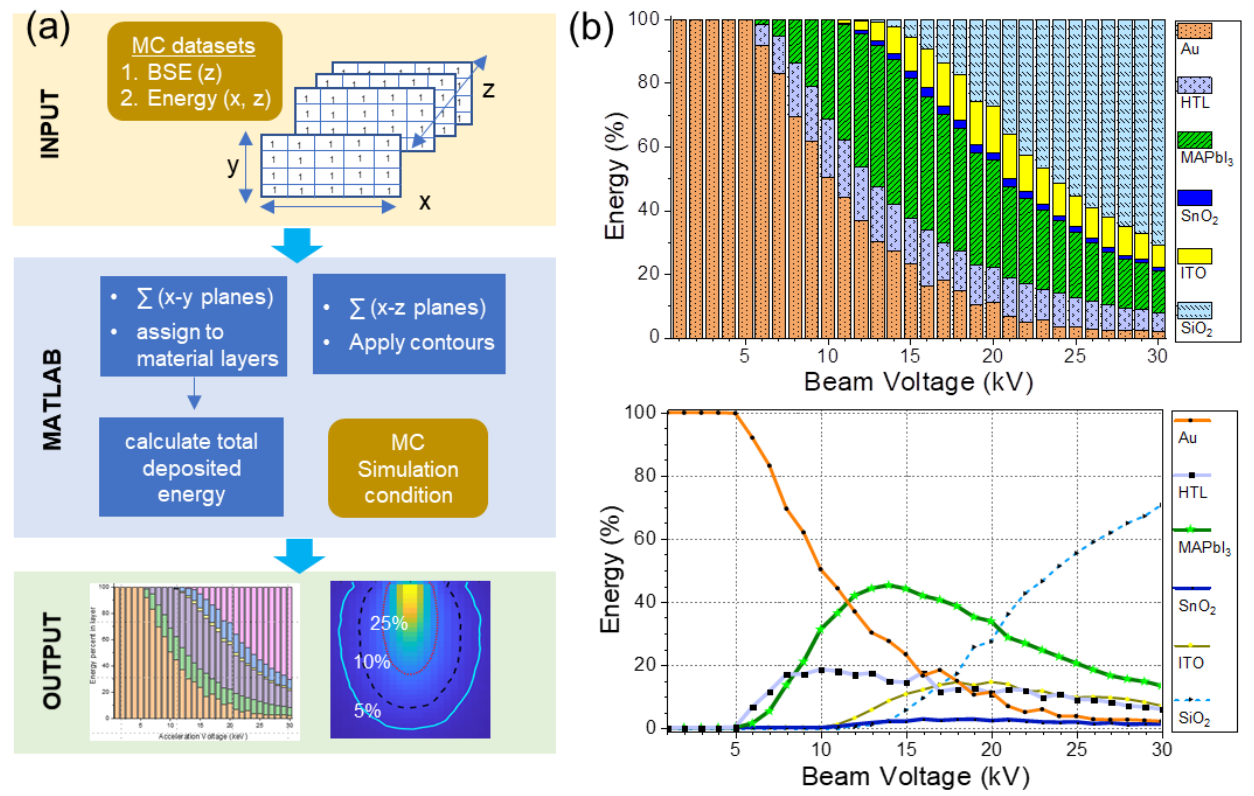

Figure 2. (a) Flowchart of MATLAB analysis program. (b) Energy deposition percentage per each layer compared to total deposited energy from the analysis of Monte Carlo simulations. 


\section{References}

[1] Ran, O. Dyck, X. Wang, B. Yang, D. B. Geohegan, and K. Xiao, "Electron-Beam-Related Studies of Halide Perovskites: Challenges and Opportunities," Adv. Energy Mater., vol. 10, no. 26, p. 1903191, Jul. 2020, doi: 10.1002/aenm.201903191.

[2] S. Chen et al., "Atomic scale insights into structure instability and decomposition pathway of methylammonium lead iodide perovskite," Nat Commun, vol. 9, no. 1, p. 4807, Dec. 2018, doi: 10.1038/s41467-018-07177-y.

[3] J. S. Manser, J. A. Christians, and P. V. Kamat, "Intriguing Optoelectronic Properties of Metal Halide Perovskites," Chem. Rev., vol. 116, no. 21, pp. 12956-13008, Nov. 2016, doi: 10.1021/acs.chemrev.6b00136. [4] R. Gauvin, E. Lifshin, H. Demers, P. Horny, and H. Campbell, "Win X-ray: A New Monte Carlo Program that Computes X-ray Spectra Obtained with a Scanning Electron Microscope," Microscopy and Microanalysis, vol. 12, no. 1, pp. 49-64, Feb. 2006, doi: 10.1017/S1431927606060089.

[5] J. Bausells, "EISS - Electron beam Monte Carlo simulator," 2008.

[6] E. Napchan, "Monte Carlo simulations of electron trajectories for samples with complex geometries," Scanning, vol. 28, pp. 111-111, Mar. 2006.

[7] D. Drouin, A. R. Couture, D. Joly, X. Tastet, V. Aimez, and R. Gauvin, "CASINO V2.42 - A fast and easyto-use modeling tool for scanning electron microscopy and microanalysis users," Scanning, vol. 29, no. 3, pp. 92-101, Jun. 2007, doi: 10.1002/sca.20000. 